\title{
What matters when judging intentionality-moral content or normative status? Testing the rational scientist model of the side-effect
}

\author{
C. Papadopoulos ${ }^{1}$ • B. K. Hayes ${ }^{1}$
}

Published online: 15 May 2017

(C) Psychonomic Society, Inc. 2017

\begin{abstract}
Previous work has demonstrated a "side-effect effect," such that intentionality is more likely to be attributed to agents who bring about negatively valenced as opposed to positively valenced side effects. The rational-scientist model explains this by suggesting that norm-violating side effects are more informative for inferring intentionality than normconforming side effects. In the present study we reexamined this account, addressing limitations of previous empirical tests (e.g., Uttich \& Lombrozo, Cognition 116: 87-100, 2010). Side-effect valence and norm status were manipulated factorially, enabling an examination of the impact of norm status on intentionality judgments in both positively and negatively valenced side effects. Additionally, the impact of sideeffect norm status on the perceived valences of side effects and agents was examined. Effects of norm status were found for both positive and negative side effects. Violation of an ostensibly neutral norm led to negative perceptions of the side effect. However, a norm status effect on intentionality judgments persisted when these effects were controlled. These results support the view that the side-effect effect is the result of the rational use of social-cognitive evidence.
\end{abstract}

Keywords Judgment and decision making · Social cognition · Causal reasoning

Human interactions are informed by moral evaluations of observed behaviors and their consequences. Of critical importance to moral evaluations is whether a person is judged to

B. K. Hayes

B.Hayes@unsw.edu.au

1 School of Psychology, University of New South Wales, Sydney, NSW 2052, Australia have acted intentionally. Traditionally, judgments about whether an agent acted intentionally were thought to arise from an assessment of factors inherent to the agent (such as mental states), with intentionality judgments serving as input to moral evaluations. This assumption is reflected in many models of intentionality (Malle \& Knobe, 1997), moral development (Turiel, 1983), and legal practice, all of which draw a sharp distinction between the evaluations of intended and unintended actions.

However, these assumptions have been challenged by the robust demonstration of a side-effect effect. In the original demonstration (Knobe, 2003), participants were presented with vignettes describing a moral agent (a company chairman or CEO) with a stated primary goal (maximizing profit). The agent's actions in pursuing this primary goal brought about a foreseen but unintended side effect. Crucially, this side effect had either a negative valence (the environment was harmed) or a positive valence (the environment was helped). Most participants judged that the negative side effect was caused intentionally, but few attributed intentionality to the positive side effect. This asymmetry has been replicated using a variety of scenarios (e.g., Cushman \& Mele, 2008; Knobe, 2010), in a range of cultures (e.g., Knobe \& Burra, 2006), and even when simplified cases are presented to 4-year-old children (Leslie, Knobe, \& Cohen, 2006). The effect is important because it suggests that attributions about the intentionality of an agent's actions are affected by the moral valence of the ostensibly unintended outcomes.

Intuitive moralist explanations of the side-effect effect (Knobe, 2010; Pettit \& Knobe, 2009) propose that theory of mind (i.e., intentionality) judgments are influenced by moral considerations. Such accounts assume that the positive or negative valence of an observed outcome is seen as a key source of information when judging whether the action that led to the outcome was intentional. In this account, morally negative 
outcomes automatically favor attributions of intentionality. Alternatively, "biased-scientist" accounts suggest that negative outcomes lead people to perceive agents as blameworthy, with blame judgments then enhancing intentionality judgments because the preceding blame response requires a justification (Adams \& Steadman, 2004; Alicke, 2008). In this sense, the valence of the observed outcomes of behavior still exerts a potentiating influence on intentionality, but the effect is less direct than that suggested by the intuitive-moralist account. That is, intuitive-moralist accounts regard moral valence as an inherent, and therefore constitutive, component of intentionality judgments, whereas biased-scientist accounts treat valence as a biasing/extraneous factor. Despite differing in detail, each of these approaches sees the moral content or valence of side effects as a key driver of intentionality judgments.

\section{A "rational" account of the side-effect effect}

A very different explanation of the side-effect effect was suggested by Uttich and Lombrozo (2010). According to their "rational-scientist" model, people use information about whether an agent's actions conform to or violate normative expectations to inform theory-of-mind judgments. The key assumption is that observed actions that conform to social norms provide a weak basis upon which to make attributions about the mental states of an agent, whereas norm-violating actions provide a strong basis for making such attributions. This asymmetry drives the side-effect effect. When someone helps the environment, they are seen to be following a widely held social norm, so that little can be inferred about their individual intentions. In contrast, harming the environment is assumed to violate social norms and hence is perceived as intentional. Whereas other accounts treat moral considerations as constitutive or biasing, the rational-scientist model treats such considerations as an evidentiary, and hence a "rational," basis for theory-of-mind judgments. According to this account, the norms in question do not have to be prescriptive or moral; the model also applies to norms that simply describe how most other people behave in a given situation.

It is important to note that other accounts also identify norm conformity and violation as important influences on intentionality judgments. Holton (2010), for example, suggests that in the harm version of the CEO scenario, the fact that the CEO has knowledge that the environment will be harmed but disregards this is a sufficient basis upon which to ascribe intentionality. In the help version, the CEO would also have to be guided by pro-environmental norms before intentionality is ascribed. Similarly, Alfano, Beebe, and Robinson (2012) proposed that norm-violating agents are more likely to reflect upon their decision (which is "rational," given the greater costs associated with violating norms) and consequently are more likely to be seen as acting intentionally. Although differing in their details, these "rational" approaches all treat the use of side-effect norm status as a rational and evidentiary basis for intentionality judgments.

According to Uttich and Lombrozo (2010), most previous tests of the side-effect effect have confounded side-effect moral valence with side-effect norm status. To avoid this confound, Uttich and Lombrozo (2010, Exp. 1) reframed the CEO scenario so that the moral valence and actual probability of the side effect was held constant (i.e., there was always a $25 \%$ chance that the environment would be harmed). The norm status of the side effect was manipulated by stipulating that the program should only proceed either if the probability of harming the environment was less than $45 \%$ (the normconforming condition) or less than $5 \%$ (the norm-violating condition). Consistent with the rational-scientist model, when the CEO adopted the program and the environment was harmed, ratings of intentionality were higher when the side effect was norm-violating than when it was norm-conforming (referred to as the norm status effect). A similar result was found when the side effect was morally neutral (i.e., whether the CEO followed or violated a convention related to the preferred color of manufactured gizmos). More recently, increased attribution of intentionality following the violation of a conventional but morally neutral norm has been demonstrated in children as young as 4 years (Rakoczy et al., 2015).

However, before the rational-scientist model can be regarded as a complete account of the side-effect effect, some limitations need to be addressed. First, Uttich and Lombrozo (2010, Exp. 1) only examined the effect of norm status on negative side effects. A strong version of their account suggests that the effect of norm conformity or violation on intentionality judgments should operate for both negative and positive outcomes. When a positive side effect comes about because of actions that violate a normative convention, this should increase the attribution that the person intentionally engaged in the behavior that led to the side effect. Uttich and Lombrozo (Exp. 2) attempted to test this prediction by constructing a "supervillain" vignette in which the norm was to take actions that result in harm. When the supervillain's actions led to a norm-violating positive side effect, there was a trend toward increased attribution ratings of intentionality. However, it was unclear whether this trend was statistically reliable. $^{1}$

In related work, Knobe (2007) presented participants with scenarios set in Nazi Germany and manipulated whether the side effect conformed to or violated an immoral "racial identification law." This created a situation in which norm violation could be perceived as morally positive. Participants were

\footnotetext{
${ }^{1}$ Uttich and Lombrozo (2010, Exp. 2) did not report a statistical test of the difference between the norm-violating help and norm-conforming help conditions.
} 
more likely to judge that an agent intentionality violated than conformed to the law. Knobe (2007) attributed these findings to "unconscious" disapproval of disobeying laws. Robinson, Stey, and Alfano (2015) expanded on this result, demonstrating a number of scenarios in which the violation of an "immoral" law was perceived as intentional.

One limitation of the Knobe (2007) and Robinson et al. (2015) results is that they can be seen as involving conflict between two opposing moral norms (e.g., obeying laws enacted by the government and courts vs. protecting the members of minority groups). In such cases, agents violate one of these norms and conform to the other. Perceptions of intentionality are then driven by violation of whichever norm is considered stronger or more salient (Robinson et al., 2015).

In contrast, the rational-scientist model suggests that violations of morally neutral conventional norms can also be informative about individual intentions. Hence, in the present study we presented numerical ranges that described conventional norms associated with positive and negative morally charged side effects, and we compared perceptions of intentionality when these norms were followed or violated. We followed this same approach with a second task in which the side effects were morally neutral. This represented a more direct test of the effect of norm conformity/violation on the perceived intentionality of both negative and positive side effects. This method also avoids the difficulty of assessing the relative salience of moral and conventional norms (cf. Robinson et al., 2015).

A second limitation of previous tests of the rationalscientist account that we addressed in the present study concerns a potential interaction between norm status and the perceived valences of the side effects and agents. The rationalscientist model assumes that norm conformity can be manipulated independently of valence. In practice, however, this may be difficult to achieve. There are good reasons to believe that at least some participants will have a metabelief that norm-violating side effects/agents are bad (or blameworthy) and that norm-conforming side effects/agents are good. Previous work shows that agents who violate conventional norms are generally held responsible for doing so (Mandelbaum \& Ripley, 2012). Moreover, from an early age, norm following is perceived positively (Rakoczy, Warneken, \& Tomasello, 2008), whereas norm violation is often seen as a form of social transgression (Turiel, 1983).

This raises the possibility that what appears to be an influence of norm violation on the side-effect effect actually reflects changes in perceived valence. Increased attributions of intentionality when norms are violated may actually be due to such violations being perceived as socially negative. Hence, in the present study we examined changes in the perceived valence of the agents and their actions as a function of norm status. We then examined whether norm status effects persisted when valence effects were controlled. In this respect, the present studies represent a more stringent test of the predictions of the rational-scientist account than previous studies. Neither Robinson et al. (2015) nor Rakoczy et al. (2015) examined the perceived valence of norm violation. Knobe (2007) asked participants to rate the blameworthiness of agents in his "Nazi race laws" scenario but did not control for this factor when analyzing intentionality. Uttich and Lombrozo (2010) examined changes in the perceived valences of side effects/agents in their "superhero" and "supervillain" scenarios, but not when conventional norms were violated.

In this study we examined the norm status effect for positively and negatively valenced side effects and for side effects with neutral valence. Toward this end, we adapted the paradigm and vignettes employed by Uttich and Lombrozo (2010, Exp. 1). Participants completed a modified version of the CEO scenario in which side-effect valence and norm conformity were manipulated factorially. According to the rationalscientist model, we should see an effect of norm status on perceived intentionality for both positive and negative side effects. When a negative side effect conforms with a stated norm, this should reduce perceived intentionality (replicating Uttich \& Lombrozo, 2010). However, when a positive side effect violates a norm, this should increase intentionality.

A second task employed the "Gizmo" vignette from Uttich and Lombrozo (2010), in which intentionality was evaluated for side effects that conformed to or violated a convention (the standard color of manufactured gizmos) that was ostensibly neutral in moral valence. Again, it was expected that perceived intentionality should be higher for norm-violating than for norm-conforming side effects. Notably, in both the morally valenced and neutral tasks, we probed for changes in perceived valence as a function of norm conformity. The rational-scientist model predicts that norm status effects on intentionality should persist over and above such shifts in valence.

\section{Method}

\section{Participants}

A total of 240 participants (136 males, 104 females) were recruited online through Amazon Mechanical Turk. Participants were drawn exclusively from the United States and were paid $\$ 0.50$ (USD). Twenty-nine participants were excluded because they failed four or more of the six attention check questions (final sample: $N=211,124$ males, 87 females; age, $M=35.6$ years, $S D=12.80)^{2}{ }^{2}$

\footnotetext{
${ }^{2}$ All of the key results reported in the main analysis (i.e., a side-effect effect for the moral vignettes and norm status effects for both the moral and neutral vignettes) were also obtained when only data from those who passed all attention checks $(n=158)$ were analyzed.
} 


\section{Design and materials}

All participants completed two tasks in counterbalanced order. The moral vignettes employed a 2 (side-effect valence: help, harm) $\times 2$ (norm status: norm-conforming, norm-violating) between-subjects design. The morally neutral vignettes employed a single factor (norm status: norm-conforming, norm-violating) between-subjects design.

The moral ("EPA") and morally neutral ("Gizmo") vignettes are shown in Figs. 1 and 2. In the moral vignettes, side-effect norm status was manipulated using invented norms that stated that a regulatory agency recommends that a program only be started if the probability of bringing about the side effect is between $10 \%$ and $50 \%$. Norm-conforming side effects fell within the normative range, whereas normviolating side effects fell outside this range. Normconforming side effects always had a $30 \%$ chance of occurring, whereas norm-violating side effects always had an associated probability of $70 \%$. The neutral vignettes were taken from Uttich and Lombrozo (2010) and employed side effects corresponding to arbitrary conventions (i.e., whether a Gizmo was dark-colored).

\section{Procedure}

Participants signed up through Amazon Mechanical Turk and were directed to the experiment hosted on Qualtrics. All participants were presented with one moral and one morally neutral vignette. Participants were randomly allocated to one of four conditions in the morally valenced task (harmful side effect, norm-conforming; harmful side effect, normviolating; helpful side effect, norm-conforming; helpful side effect, norm-violating). They were allocated to the corresponding norm status condition for the morally neutral task.

For each vignette, after receiving general instructions, participants were presented with the vignette text on screen. Reading the vignette was self-paced, with the restriction that the participant could not commence the question stage of the task until at least $35 \mathrm{~s}$ had elapsed. Participants were then presented with three comprehension/attention check items (e.g., "After the program was started by the CEO what happened to the environment?").

These were followed by a series of test questions. The first was a rating of perceived intentionality ("How appropriate is it to say that the CEO intentionally helped/harmed the environment?" 1 = not at all appropriate, 7 = very appropriate).

EPA Vignette

The Environmental Protection Authority (EPA) is a regulatory agency that aims to set

environmental standards for the energy industry even though it does not have the authority

to enforce compliance with these standards. The EPA has established a standard that an

energy company should only start a new industrial program if the chance of the program

helping [harming] the environment (e.g. through the production of clean energy) [e.g.

through the production of pollution] is in the range of $10-50 \%$.

The vice-president of an energy production company went to the CEO and said, "We are

thinking of implementing a new program. It will help us increase profits and the chance

that it will help [harm] the environment is $30 \% \mathbf{( 7 0 \% )}$. The industry standard is to only

start programs of this type if the probability of helping [harming] the environment is in the

range of $10-50 \%$. Therefore, we would be complying with the industry standard

(violating the industry standard).

The CEO said, "I don't care at all about helping [harming] the environment. I just want to

make as much profit as I can. Let's start the new program".

They started the new program. As it happened, the environment was helped [harmed].

Fig. 1 The moral vignette. The main text corresponds to the help/norm-conforming condition. Text in the square brackets/italics shows the instructions for the corresponding harm condition. Text in parentheses/bold font shows the instructions for the corresponding norm-violating condition 
Gizmo Vignette

The Gizmo Aesthetics Standards Authority (GASA) is a regulatory body that aims to

provide some industrial standards for the gizmo industry even though it does not have the

authority to enforce compliance with these standards. These standards are related to the

physical appearance of gizmos in order to avoid gizmos being confused with different

devices that may appear similar. The GASA has established a standard that a gizmo

company should only start a new program if the chance of the program creating dark-

colored gizmos (which can be confused with different devices that are generally dark-

colored) is in the range of $10-50 \%$.

The vice-president of a gizmo company went to the CEO and said, "We are thinking of

implementing a new program. It will help us increase profits and the chance that it will

result in dark-colored gizmos is $30 \% \mathbf{( 7 0 \% )}$. The industry standard is to only start

programs of this type if the probability of creating dark-colored gizmos is in the range of

$10-50 \%$. Therefore, we would be complying with the industry standard (violating the

industry standard).

The CEO said, "I don't care at all about the colour of the gizmos. I just want to make as

much profit as I can. Let's start the new program”.

They started the new program. As it happened, the gizmos were dark-colored.

Fig. 2 The neutral vignette. The main text corresponds to the norm-conforming condition. Text in parentheses/bold font shows the instructions for the corresponding norm-violating condition

This was followed by ratings of the valences of the side effect ("To what extent do you think that the environment being helped/harmed was good or bad?" $1=$ bad, $7=$ good ) and the agent ("To what extent do you think that the CEO is a good or a bad person?" $1=\mathrm{bad}, 7=\mathrm{good}$ ). The final question queried the extent to which the agent deserved to be praised for the actions ("To what extent do you think that the CEO should be blamed or praised?" 1 = blamed, 7 = praised ). After participants had completed these questions, the second vignette was presented.

\section{Results}

There was no effect of gender or task order on the intentionality ratings for moral vignettes or neutral vignettes, $F_{\mathrm{S}}<2.8$. Hence, all analyses were collapsed across these factors.

\section{Moral vignettes}

The first analyses checked the effectiveness of the valence manipulation. As expected, participants perceived the harmful side effect less positively $(M=1.42, S D=$ $0.89)$ than the helpful side effect $(M=6.42, S D=$ $1.10), F(1,207)=1,301.21, p<.001, \eta_{\mathrm{p}}^{2}=.86$. Likewise, agents were perceived less positively in the harm $(M=2.31, S D=1.05)$ than in the help $(M=$ $3.08, S D=1.36)$ condition, $F(1,207)=22.03, p<$ $.001, \eta_{\mathrm{p}}{ }^{2}=.10$. Agents in the harm condition were rated as being less deserving of praise $(M=1.87, S D$ $=1.03)$ than those in the help condition $(M=4.04, S D$ $=0.95), F(1,207)=254.64, p<.001, \eta_{\mathrm{p}}{ }^{2}=.55$. These results confirm the effectiveness of our manipulation of side-effect valence. No differences between the normconforming and norm-violating conditions were found on any of these ratings (all $F_{\mathrm{S}}<1.0$ ).

The intentionality ratings for each experimental condition are shown in Fig. 3. These data were entered into a 2 (valence) $\times 2$ (norm status) analysis of variance (ANOVA). The main effect of side-effect valence was significant, $F(1,207)=$ $143.37, p<.001, \eta_{\mathrm{p}}{ }^{2}=.41$. Intentionality ratings were higher in the harm $(M=4.42, S D=1.99)$ than in the help $(M=1.80$, $S D=1.19)$ condition, consistent with the side-effect effect. 


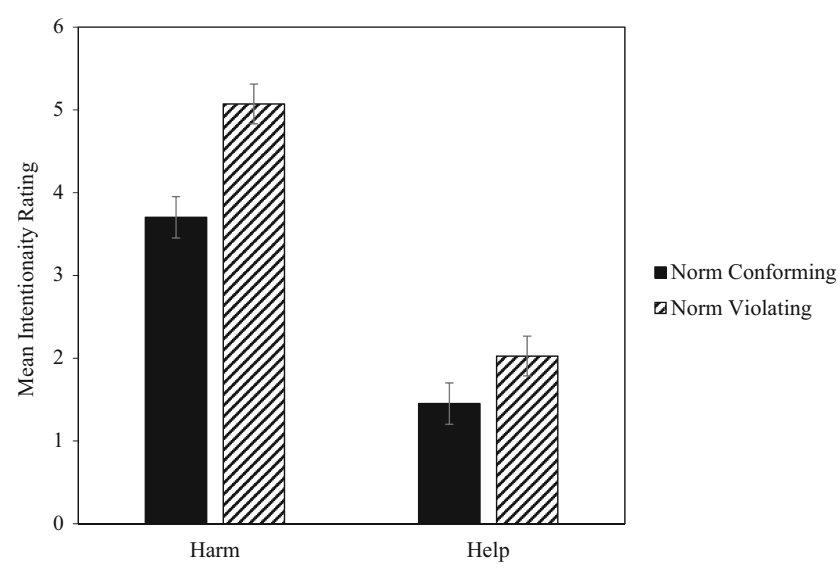

Fig. 3 Mean intentionality ratings for the moral vignette

The main effect of norm status was also significant, $F(1,207)$ $=12.48, p=.001, \eta_{\mathrm{p}}{ }^{2}=.06$. Intentionality ratings were higher in the norm-violating $(M=3.50, S D=2.23)$ than in the normconforming $(M=2.73, S D=1.88)$ condition, consistent with the norm status effect. Notably, no interaction was found between side-effect valence and norm status, $F(1,207)=1.04, p$ $=.31$, indicating that norm status affected intentionality judgments in both the help and harm conditions. Simple-effects tests confirmed the existence of norm status effects in both the harm, $t(102)=2.63, p=.01$, and the help, $t(105)=2.45, p=$ .02 , conditions.

\section{Neutral vignettes}

Uttich and Lombrozo (2010) assumed that the normconforming and norm-violating vignettes using the Gizmo scenario would be equivalent in terms of moral valence. However, our valence check questions challenged this assumption, with violation of the conventional norm being perceived less positively $(M=3.27, S D=$ 1.33) than norm conformity $(M=4.07, S D=1.54), F(1$, 209) $=16.35, p<.001, \eta_{\mathrm{p}}{ }^{2}=.07$. Norm-violating agents tended to be viewed less positively $(M=3.18, S D=1.36)$ than norm-conforming agents $(M=3.45, S D=1.03)$, but this trend did not reach significance, $F(1,209)=2.64, p=$ .10. Norm violators were seen as less deserving of praise $(M=3.04, S D=1.41)$, than norm conformers $(M=3.49$, $S D=1.11), F(1,209)=6.76, p=.01, \eta_{\mathrm{p}}{ }^{2}=.03$.

Because norm violation was viewed more negatively than norm conformity, side-effect valence was included as a covariate in the analysis of intentionality ratings for the neutral vignette. Notably, side effects that were normviolating were judged as being more intentional $(M=3.7$, $S D=1.91)$ than side effects that were norm-conforming $(M=2.7, S D=1.69)$, even after adjusting for the valence covariate, $F(1,208)=11.25, p=.001, \eta_{\mathrm{p}}{ }^{2}=.05$. For the neutral vignettes, the effect of norm status on intentionality persisted when the effect of valence was controlled. ${ }^{3}$

\section{Discussion}

Uttich and Lombrozo (2010) advanced a novel explanation of the side-effect effect, suggesting that it is due to increased attribution of intentionality when a side effect violates either moral or conventional norms. In the present study, we examined further evidence for this claim. For the moral vignettes we found a robust side-effect effect, with harmful side effects generally being perceived as more likely to have been caused intentionally than helpful side effects (cf. Cushman \& Mele, 2008; Knobe, 2010). Like Uttich and Lombrozo (2010) and Rakoczy et al. (2015), we found that for harmful side effects, attributions of intentionality were reduced when the side effect conformed with a stated norm. An important result, however, was that a symmetrical effect was obtained for attributions of intentionality for positive side effects. Positive side effects were viewed as being more intentional when they violated a norm than when they were norm conforming.

An even clearer demonstration of the informative role of norm following or violation in ascribing intentionality to an agent comes from our examination of norm status in ostensibly "neutral" vignettes. There were two important results. First, although the side effects were not explicitly described as harmful or helpful, the side effects and the agents who enacted them were both viewed more negatively when they violated a stated norm than when they conformed with the norm. This result highlights the pervasive tendency to view norm conformity as being socially positive (cf. Rakoczy et al., 2008; Turiel, 1983). The second and most notable result was that norm status affected attributions of intentionality even when the effects of valence were controlled. Side effects that violated a conventional norm were viewed as more likely to have been brought about intentionally than those conforming to a norm.

These results are consistent with the rational-scientist account of the side-effect effect. In both types of vignettes, engaging in behavior that led to a violation of a stated norm was seen as evidence of individual intention, whereas attributions of intentionality were lower when the side-effect outcome was normconforming. Our findings, however, extend the rational-scientist account by (a) showing that the effects of norm status on perceived intentionality apply to positive side effects, and (b)

\footnotetext{
${ }^{3}$ The difference in intentionality was maintained when the covariate was not included, $F(1,209)=16.10, p<.001, \eta_{\mathrm{p}}{ }^{2}=.07$. As a further test of the effect of norm status, a multiple regression was carried out on intentionality judgments with the valence measures for each condition and norm status entered on separate steps. Norm status remained a reliable predictor of intentionality, even when it was added to the regression model after valence, $\Delta R^{2}=.08, F(1,208)$ $=11.25, p=.001$.
} 
clarifying that an effect of norm status on intentionality in neutral vignettes exists even when valence effects are controlled.

These results challenge intuitive-moralist accounts that suggest that the side-effect effect is the result of an inherent potentiating effect of moral valence on judgments of intentionality (Knobe, 2010; Pettit \& Knobe, 2009). Such accounts have difficulty explaining why norm status affected the intentionality judgments associated with both positive and negative side effects. They also have difficulty explaining the existence of a norm status effect in neutral scenarios when valence effects are controlled. Likewise, the finding of a robust norm status effect in the neutral scenario suggests that the side-effect effect involves more than a pragmatic or motivational (blamebased) bias on the interpretation of intentionality questions (cf. Adams \& Steadman, 2004; Alicke, 2008).

The rational-scientist model, supported by our study, offers a new interpretation of empirical findings that have previously been explained using an intuitive-moralist framework. For example, Knobe (2004) presented a side effect involving a CEO making movies better or worse from an artistic standpoint, and found that intentionality attributions were stronger in the latter case. Intuitive-moralist accounts explain this result by assuming that making bad movies is evaluated as a negative outcome and that this valenced evaluation influences intentionality judgments. Arguably, the rational-scientist approach offers a more direct explanation of such effects by suggesting that making bad movies violates an industry norm and, hence, is likely to be viewed as the result of an intentional act.

The present findings are compatible with other "rational" approaches to the side-effect effect. For example, Holton (2010) and Robinson et al. (2015) also predicted greater intentionality attributions for norm-violating side effects in the case of prescriptive norms. However, unlike Holton, the rationalscientist model predicts intentionality asymmetries as a function of norm status for both prescriptive norms (either moral, such as helping the environment, or conventional, such as making gizmos of a certain color) and descriptive norms (e.g., the percentage of people who frequent a local cafe). Moreover, the present study goes beyond these previous studies to show an influence of side-effect norm status on intentionality judgments over and above changes in the perceived valence of the side effects.

Although the present findings speak against the intuitivemoralist account of the side-effect effect, a modified version of that account may be viable. Knobe (2010) proposed that the impact of moral considerations on intentionality judgments is mediated by default attitudes toward the side effect. People expect an agent to be opposed to negative side effects and in favor of positive side effects. When the agent expresses indifference toward the side effect, people tend to perceive the agent as being in favor of the negative but not the positive side effect. Although Knobe (2010) proposes that a priori differences in moral beliefs influence perceptions of an agent's attitudes, it is possible that the norm status of a side effect could also exert an influencethat is, people may view norm following as a positively valenced default, as compared with norm violation. Hence, although it would be a greater stretch for intuitive-moralist approaches than for the rational-scientist model, it is possible that ad hoc adjustments could be made to the former to accommodate the present findings.

The present results do not rule out the possibility of an additional effect of moral valence on judgments of intentionality, over and above the effect of norm status. The large difference in intentionality judgments for the harm and help vignettes could be due to strong norms favoring actions that lead to positive environmental outcomes, as was claimed by Uttich and Lombrozo (2010), to the constitutive (Knobe, 2010) or biasing (Adams \& Steadman, 2004; Alicke, 2008) effect of valence, or to some combination of these factors. It remains for future research to separate out the contributions of these factors to judgments of intentionality. What is not in dispute, however, is that the norm status of observed behavioral outcomes has a robust effect on how people evaluate individuals' intentionality, and that this can explain the asymmetry in intentionality ratings observed in the side-effect effect.

Author note This work was supported by an Australian Postgraduate Award to the first author and by Australian Research Council Discovery Grant DP13010447 to the second author.

\section{References}

Adams, F., \& Steadman, A. (2004). Intentional actions and moral considerations: Still pragmatic. Analysis, 64, 268-276.

Alfano, M., Beebe, J. R., \& Robinson, B. (2012). The centrality of belief and reflection in Knobe-effect cases: A unified account of the data. The Monist, 95, 264-289.

Alicke, M. D. (2008). Blaming badly. Journal of Cognition and Culture, 8 , $179-186$.

Cushman, F., \& Mele, A. (2008). Intentional action: Two-and-a-half folk concepts? In J. Knobe (Ed.), Experimental philosophy (pp. 171188). Oxford, UK: Oxford University Press.

Holton, R. (2010). Norms and the Knobe effect. Analysis, 70, 417-424.

Knobe, J. (2003). Intentional action and side effects in ordinary language. Analysis, 63, 190-193.

Knobe, J. (2004). Folk psychology and folk morality: Response to critics. Journal of Theoretical and Philosophical Psychology, 24, 270-279.

Knobe, J. (2007). Reason explanation in folk psychology. Midwest Studies in Folk Psychology, 31, 90-106.

Knobe, J. (2010). Person as scientist, person as moralist. Behavioral and Brain Sciences, 33, 315-365.

Knobe, J., \& Burra, A. (2006). The folk concept of intention and intentional action: A cross-cultural study. Journal of Cognition and Culture, 6, 113-132.

Leslie, A. M., Knobe, J., \& Cohen, A. (2006). Acting intentionally and the side-effect effect: Theory of mind and moral judgment. Psychological Science, 17, 421-427.

Malle, B. F., \& Knobe, J. (1997). The folk concept of intentionality. Journal of Experimental Social Psychology, 33, 101-121. 
Mandelbaum, E., \& Ripley, D. (2012). Explaining the abstract/concrete paradoxes in moral psychology: The NBAR hypothesis. Review of Philosophy and Psychology, 3, 351-368.

Pettit, D., \& Knobe, J. (2009). The pervasive impact of moral judgment. Mind and Language, 24, 586-604.

Rakoczy, H., Warneken, F., \& Tomasello, M. (2008). The sources of normativity: Young children's awareness of the normative structure of games. Developmental Psychology, 44, 875-881.

Rakoczy, H., Behne, T., Clüver, A., Dallmann, S., Weidner, S., \& Waldmann, M. (2015). The side-effect effect in children is robust and not specific to the moral status of action effects. PLoS One, 10(e132933), 1-10. doi:10.1371/journal.pone. 0132933

Robinson, B., Stey, P., \& Alfano, M. (2015). Reversing the side-effect effect: The power of salient norms. Philosophical Studies, 172, 177-206.

Turiel, E. (1983). The development of social knowledge: Morality and convention. Cambridge, UK: Cambridge University Press.

Uttich, K., \& Lombrozo, T. (2010). Norms inform mental state ascriptions: A rational explanation for the side-effect effect. Cognition, $116,87-100$ 